\title{
End-to-End simulations for the MICADO-MAORY SCAO mode
}

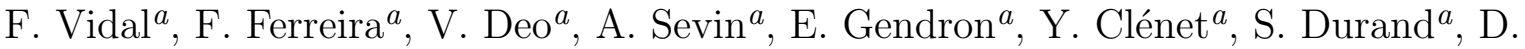

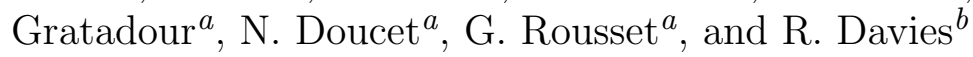 \\ ${ }^{a}$ Laboratoire d'Etudes Spatiales et d'Instrumentation en Astrophysique (LESIA), \\ Observatoire de Paris, CNRS, UPMC, Universite Paris Diderot; 5 Place Jules Janssen, 92190 \\ Meudon, France \\ ${ }^{b}$ Max-Planck-Institut fur Extraterrestrische Physik, Garching, Germany
}

\begin{abstract}
MICADO is a E-ELT first light near-infrared imager. It will work at the diffraction limit of the telescope thanks to multi-conjugate adaptive optics (MCAO) and single-conjugate adaptive optics (SCAO) modes provided inside the MAORY AO module. The SCAO capability is a joint development by the MICADO and MAORY consortia, lead by MICADO, and is motivated by scientific programs for which SCAO will deliver the best AO performance (e.g. exoplanets, solar system science, bright AGNs, etc). Shack-Hartmann (SH) or Pyramid WFS were both envisioned for the wavefront measurement of the SCAO mode. In addition to WFS design considerations, numerical simulations are therefore needed to trade-off between these two WFS. COMPASS (COMputing Platform for Adaptive optics SyStems) is a GPU-based adaptive optics end-to-end simulation platform allowing us to perform numerical simulations in various modes (SCAO, LTAO, MOAO, MCAO...). COMPASS was originally bound to Yorick for its user interface and a major upgrade has been recently done to now bind to Python allowing a better long term support to the community. Thanks to the speed of computation of COMPASS we were able to span quickly a very large parameters of space at the E-ELT scale. We present the results of the study between WFS choice (SH or Pyramid), WFS parameters (detector noise, guide star magnitude, number of subapertures, number of controlled modes...), turbulence conditions and AO controls for the MICADO-MAORY SCAO mode.
\end{abstract}

Keywords: SCAO, Pyramid wavefront sensor, E-ELT

\section{INTRODUCTION}

MICADO is the first light near-IR camera $(0.9-2.5 \mu \mathrm{m})$ on the E-ELT. It has been designed to work at the diffraction limit over a 1' field of view (Davies et al. $2016^{1}$ ) and will come with a long slit spectroscopic mode, at a moderate spectral resolution (5000 to 10000). The Adaptive Optics (AO) correction is supplied by the Multi Conjugate Adaptive Optics (MCAO) module called MAORY (Diolaiti et al. $2016^{2}$ ) . For full scientific exploitation and in a phased approach for developing AO performance at the E-ELT, a SCAO mode is needed for MICADO. It is a joint development between the MICADO and MAORY consortia, integrated into MAORY. This SCAO module will use the built-in deformable mirror (DM) of the E-ELT, the so-called M4 mirror, and a dichroic will send the visible light to the SCAO WaveFront Sensor (WFS). The MCAO module will provide a moderately high Strehl ratio over the entire 75 " field of view while the SCAO mode will provide better on-axis Strehl ratio. We focus in this paper on the latest numerical simulations dedicated to the SCAO mode.

\section{SIMULATION PARAMETERS}

We present in this section the simulation parameters we used for the results presented in section 5 . We mainly performed simulations on the Pyramid WFS since it less well known than the Shack-Hartmann. However section 5.1 also presents a comparison of performance between a Pyramid WFS and a Shack-Hartmann.

Further author information: Vidal Fabrice E-mail: fabrice.vidal@obspm.fr, Telephone: +33 (0)1 45077632 


\subsection{Turbulence parameters}

The simulated turbulence conditions were derived from the ESO specifications and computed at $30^{\circ}$ of zenith and summarized in Table 1. Five atmospheric conditions were simulated, each with a different seeing, wind speed and $\mathrm{Cn} 2$ profile spread in 35 different layers (see Fig. 1). The outer scale $\left(L_{0}\right)$ was set to $25 \mathrm{~m}$ for all layers and turbulence conditions.

\begin{tabular}{|c|clcc|c|}
\hline Conditions & Seeing (") & $\mathrm{r} 0(\mathrm{~cm})$ & $v_{0}(\mathrm{~m} / \mathrm{s})$ & $\tau_{0}(\mathrm{~ms})$ & \# Layers \\
\hline Q1 & 0.471 & 21.47 & 9.1 & 8.08 & 35 \\
Q2 & 0.619 & 16.33 & 9.13 & 6.12 & 35 \\
Median & 0.702 & 14.4 & 9.21 & 5.35 & 35 \\
Q3 & 0.793 & 12.75 & 9.13 & 4.78 & 35 \\
Q4 & 1.136 & 8.9 & 9.79 & 3.71 & 35 \\
\hline
\end{tabular}

Table 1: Turbulence condition parameters

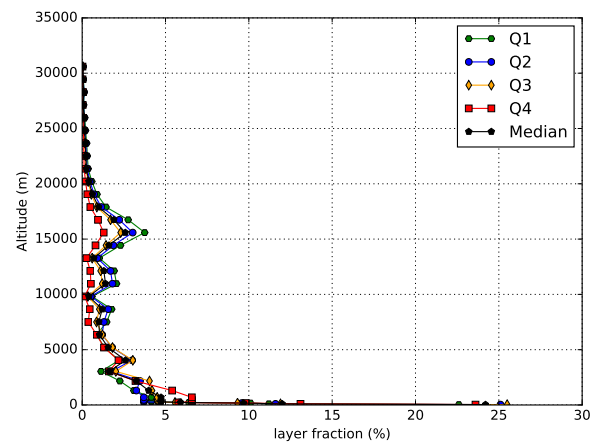

Figure 1: Cn2(h) profiles illustrarated for each of the 5 simulated conditions.

\subsection{Telescope parameters}

The E-ELT has a very specific non-telecentric pupil. Depending on the field of view needed, the shape and size of the output pupil varies. The simulated pupil for the MICADO-SCAO simulations is hexagonal and has a diameter of $38.542 \mathrm{~m}$ with a central obscuration of 0.28 . The width of the E-ELT spiders is very large and higher than the E-ELT deformable mirror (M4) equivalent pitch. This particularity leads to a non-continuity of phase not measured by the wavefront sensor. During our first tests, in presence of such a spider we observed the so-called Island effect ${ }^{4}$ due to pistons on the corrected phase for each of the 6 petals of the pupil impacting very strongly the corrected PSF. The exact impact on the presence of spiders is currently under investigation but several strategies are envisioned to overcome this problem ${ }^{5}$ (slaving actuators, filtering differential pistons in the actuators space ect...). To avoid temporarily the Island Effect in our simulation we projected on the WFS the E-ELT pupil but without the spiders. However the final PSF on target(s) was computed with the spiders to simulate the (real) diffraction effects on the final image.

\subsection{Deformable mirror parameters}

The so called M4 Unit ${ }^{3}$ provides a real-time wavefront correction capability to the E-ELT. The SCAO will use this deformable mirror for AO correction. M4 is made of 6 different petals and has a total of 5190 actuators. However a large amount of actuators are hidden by the outer edge pupil + central obscuration. In our case, with a fixed on-axis correction, the (useful) total number of actuators simulated is 4576 .

The M4 actuators geometry is non-squared. It has been reproduced carefully and placed on the E-ELT pupil accordingly to the ESO specifications. We used Schwartz influence functions having the good property to 
combine a fast descent to zero combined with a Fourier transform fully defined in the same support. This allowed us to create fully independent influence functions in much smaller support than the pupil size (for memory usage) and avoid artificial artifacts in the PSF image. Finally, the Tip-Tilt is made on a separated mirror that produce to pure TipTilt and emulate the M5 Mirror.

\subsection{WFS parameters}

One of the main study was to compare the 2 types of WFS envisioned for the SCAO: Pyramid or ShackHartmann. Given the requirements specifications of loop frequency of $500 \mathrm{~Hz}$ and the very large number of pixels required, the Shack-Hartmann requires (in its traditional dimensioning) a much larger detector than the Pyramid one. In addition, Pyramid WFS has already demonstrated its advantage over the SH, in particular, in terms of magnitude limit expected between 1 and 2 magnitude $^{6,7}$ at the E-ELT. The results of this performance comparison is presented in section 5.1. The MICADO SCAO WFS wavelength bandwidth range from 0.589 to $0.965 \mu \mathrm{m}$ but the simulated wavelength was monochromatic centered at $0.7 \mu \mathrm{m}$.

\subsubsection{Pyramid WFS}

Pyramid WFS uses 4 pupils for the $\mathrm{x}$ and $\mathrm{y}$ gradient measurement and therefore uses only 4 pixels per subaperture. The so called ALICE CCD detector (a.k.a OCAM ${ }^{8}$ ) has only 0.1e- Read Out Noise (RON) at 500Hz. With a CCD size of $240 \times 240$ this detector allows to form 4 image of the pupil with up to $120 \times 120$ pixels maximum each. Table 2 summarize the Pyramid WFS parameters we used during our simulations. Figure 2 illustrates the simulated pupils formed with the Pyramid WFS.

\begin{tabular}{|c|c|}
\hline Detector size & $240 \times 240$ Pixels \\
\hline Simulated Size for the PYR image & $512 \times 512$ (used $240 \times 240$ for slopes computation $)$ \\
\hline Nb of sub-apertures (pixels per pupil) & $92 \times 92($ tested from $80 \times 80$ to $112 \times 112)$ \\
\hline RON & $0.1 \mathrm{e}-$ \\
\hline Modulation size & $3 \lambda / \mathrm{D}$ (tested from 1 to $20 \lambda / \mathrm{D})$ \\
\hline Nb of points per modulation circle & 20 at $3 \lambda / \mathrm{D}$ up to 128 at $20 \lambda / \mathrm{D}$ \\
\hline
\end{tabular}

Table 2: Baseline parameters for the simulated Pyramid WFS based on the ALICE detector.

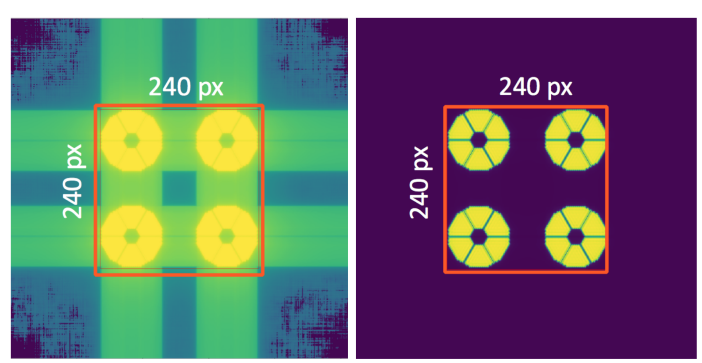

Figure 2: Simulated pupils formed on the CCD when using a Pyramid WFS (left: Log scale, right: normal scale)

The Pyramid WFS simulated algorithm takes care of the diffracted light on the edges of the pyramid and reproduce the contamination of light across the 4 pupils. The signal is computed for each sub-aperture with the following formulae:

$$
\begin{aligned}
& S_{x}(x, y)=\left[\left(I_{1}(x, y)+I_{2}(x, y)-\left(I_{3}(x, y)+I_{4}(x, y)\right)\right)\right] / I_{0} \\
& S_{y}(x, y)=\left[\left(I_{1}(x, y)+I_{4}(x, y)-\left(I_{2}(x, y)+I_{2}(x, y)\right)\right)\right] / I_{0}
\end{aligned}
$$

where $I_{i}(x, y)$ is the intensity in the sub-aperture located at $(\mathrm{x}, \mathrm{y})$ coordinates in the quadrant $i$, integrated during the entire modulation cycle and $I_{0}$ is the average intensity computed on the 4 pupils. 
Finally the gradients $G_{x}$ and $G_{y}$ are computed from the relation:

$$
\begin{aligned}
& G_{x}(x, y)=r M o d \times \sin \left(\frac{\pi}{2} S_{x}(x, y)\right) \\
& G_{y}(x, y)=r M o d \times \sin \left(\frac{\pi}{2} S_{y}(x, y)\right)
\end{aligned}
$$

where $r M o d$ is the modulation radius size expressed in arcseconds.

\subsubsection{Shack-Hartmann WFS}

Here we describe the SH specific parameters we used for the SH Vs Pyramid comparison (results presented in Section 5.1). Our preliminary simulations concluded with an optimal number of pixels of 8 pixels per subaperture. Given the required number of subaperture of at least $78 \times 78$ it leads to a much larger CCD detector than the Pyramid solution. The current most suitable imager is the LISA detector (a.k.a NGSD ${ }^{9}$ ). However this detector has a $3 \mathrm{e}-\mathrm{RON}$ at $500 \mathrm{~Hz}$. Table 3 summarize the AO parameters of the SH WFS type.

\begin{tabular}{|c|c|}
\hline Detector size (Pixels) & $840 \times 880$ \\
\hline \# sub-apertures & $78 \times 78$ \\
\hline Pixels per sub-ap. & 8 \\
\hline RON & $3 \mathrm{e}-$ \\
\hline
\end{tabular}

Table 3: Baseline parameters for the simulated Shack-Hartmann WFS based on the LISA detector.

\subsection{AO parameters}

Unless specified in the following sections the relevant AO parameters for the baseline simulations are presented in Table 4.

\begin{tabular}{|c|c|}
\hline Total Number of slopes & 12104 \\
\hline Number of actuators & $4576+2$ (Tip Tilt) \\
\hline Number of Controlled modes & 4473 \\
\hline Loop Frequency & $500 \mathrm{~Hz}$ \\
\hline Total throughput & 0.5 \\
\hline Zero point (R Band) & $2.6 \mathrm{e}^{10}$ photons $/ \mathrm{s} / \mathrm{m}^{2}$ \\
\hline Number of simulated AO iterations & 8096 \\
\hline Simulated exposure & $\approx 16$ seconds \\
\hline
\end{tabular}

Table 4: Summary of the relevant AO parameters for the SCAO simulations.

\section{SIMULATION ARCHITECTURE}

The GPU enhanced COMPASS platform ${ }^{11}$ was used to perform the E-ELT scaled SCAO simulations. Taking advantage of the specific hardware architecture of the GPU, the COMPASS tool allows to achieve adequate execution speeds to conduct large simulation campaigns for the design of the E-ELT instruments. It is based on an open source software package including some core codes in CUDA, combined with a layer of higher-level code in $\mathrm{C}++$ directly interfaced to Python.

The entire SCAO simulations results presented in this paper were performed using 1 computer equipped with 2 CPU Intel(R) Xeon(R) CPU E5-2630 v4 @ 2.20GHz (10 cores each) with 64GB RAM and 8 NVIDIA Titan X (Pascal) GPUs cards. The total cost of this machine is $15 \mathrm{kEuros}$.

The Simulation main outputs are (non-exhaustive list): 
- Long and short exposure PSFs in J, H, K bands.

- SR measurement for each PSF (Short and Long Exposure)

- Circulars buffers for slopes and commands (for post processing analysis).

- Residual error in the mirror basis computed for each controlled mode at each iteration.

- Images of the WFS detector.

- Modal gains per mirror mode.

- Modal interaction and command Matrix

- ...

The standard case runs at $10 \mathrm{~Hz}$ (SCAO using Pyramid@ 5 $/ D$ and Q3 turbulence conditions). The total simulation time to compute the full calibration procedure (see section 4 ) + the equivalent 16 seconds exposure time is $40 \mathrm{mn}$. The whole simulation is scripted to explore the desired space parameters and produce the results presented in section 5. All the simulation parameters are saved in a database and PSFs, circular buffers, interaction matrices... are stored in . fits files with fully tagged headers. A web server is also available for post processing and greatly simplifies the identification of the best parameters during space parameters exploration (data mining/ big data)

\section{CALIBRATION PROCEDURE}

We summarize here the calibration procedure we followed to optimize the AO loop residuals when using a Pyramid WFS.

The first step is to compute the mirror modes using the knowledge of the influence function shape and the actuators distribution on the pupil. We derive the mirror modes from the diagonalization of the actuators geometric covariance filtered by Tip-Tilt and piston. Therefore these modes are fixed with the E-ELT pupil and DM whatever the turbulence conditions or the WFS dimensioning. This was very helpful to determine and compare mode per mode the SCAO residual performance while spanning the whole space parameters. Note that we also tested the Zernikes and Krahunene-Loeve basis but the best results were found using the mirror modes.

The second step is to compute the modal control matrix on diffraction limited source. Here each mirror mode is produced by the DM and shown to the Pyramid WFS to measure the modal interaction matrix $\left(M_{D i f f}\right)$ on a diffraction limited source. To ensure the modes are measured in the linearity range of the Pyramid we measure the linearity response of the modes versus their amplitude. We measured the linearity range of each mode and deduce their optimal amplitude. The optimal amplitude was found to be small (less than $50 \mathrm{~nm}$ rms) ensuring to be in less than $50 \%$ range of the linear response of the Pyramid. Same procedure was performed for the TT. The interaction matrix is then truncated to keep the desired number of controlled modes and inverted to compute the modal command matrix. We typically filter 100 modes but a separate study was performed to optimally find the best number of filtered modes while exploring the SNR conditions (see section 5.3.3).

Next step is to perform a pre-convergence of the loop. We close the loop on turbulence (integrator gain of 1, modal gains all set to 1). It is crucial to realize that with the turbulence the Pyramid WFS has a modal centroid gain (also named optical gain) that depends on the amplitude and shape of the residual correction of the loop (the PSF at the top a the Pyramid is typically 5-20\% SR in V Band in our case). This sub-optimal case is enough to ensure a stable pre convergence of the loop with decent correction. We choose here to wait 100 iterations for the loop to converge onto this 1st order of correction (to sharpen the PSF on the Pyramid).

To improve the loss of performance due to the underestimated optical gain, we measure (loop closed) an on-sky modal interaction matrix $\left(M_{R e s}\right)$. This method was already proposed by Korkakioski et al. $(2007)^{10}$. It allows to improve the AO performance and loop stability especially when the SR on top of the Pyramid is low. This can be due to a WFS design choice (where the WFS wavelength is particularly short), when the number of actuators is low (leading to high wavefront residuals) or when the seeing conditions degrades. 
We used the following relation to retrieve the Pyramid modal gains:

$$
\text { Gains }=\sqrt{\frac{\operatorname{Diag}\left(M_{\text {Diff }} M_{\text {Diff }}^{t}\right)}{\operatorname{Diag}\left(M_{\text {Res }} M_{\text {Res }}^{t}\right)}}
$$

It is to be noted that these gains depends on the initial seeing, the quality of correction of the loop, the modulation radius ect... Once the modal gains are computed we apply them on the diffraction limited interaction matrix $\left(M_{D i f f}\right)$ to compute a new optimal command matrix and close the loop again.

Once the modal gains are optimized we finally use a global integrator loop optimization to deal with bandwidth error and noise propagation (I.e we find best gain to optimize SR/residual error). Please note that the last 2 steps should be iterative since the increase of performance leads to a sharpen PSF at the top of the pyramid and modifies the modal centroid gains. However we found that between 1 and 2 iterations was close to optimal.

Figure 3 shows an example of the measured modal gains and how it behaves when we change the modulation radius and residuals of loop correction (by increasing the initial seeing). The test case uses a $18 \mathrm{~m}$ diameter telescope and 40x40 actuators DM (cartesian grid) and a pyramid WFS with 45x45 subapertures.
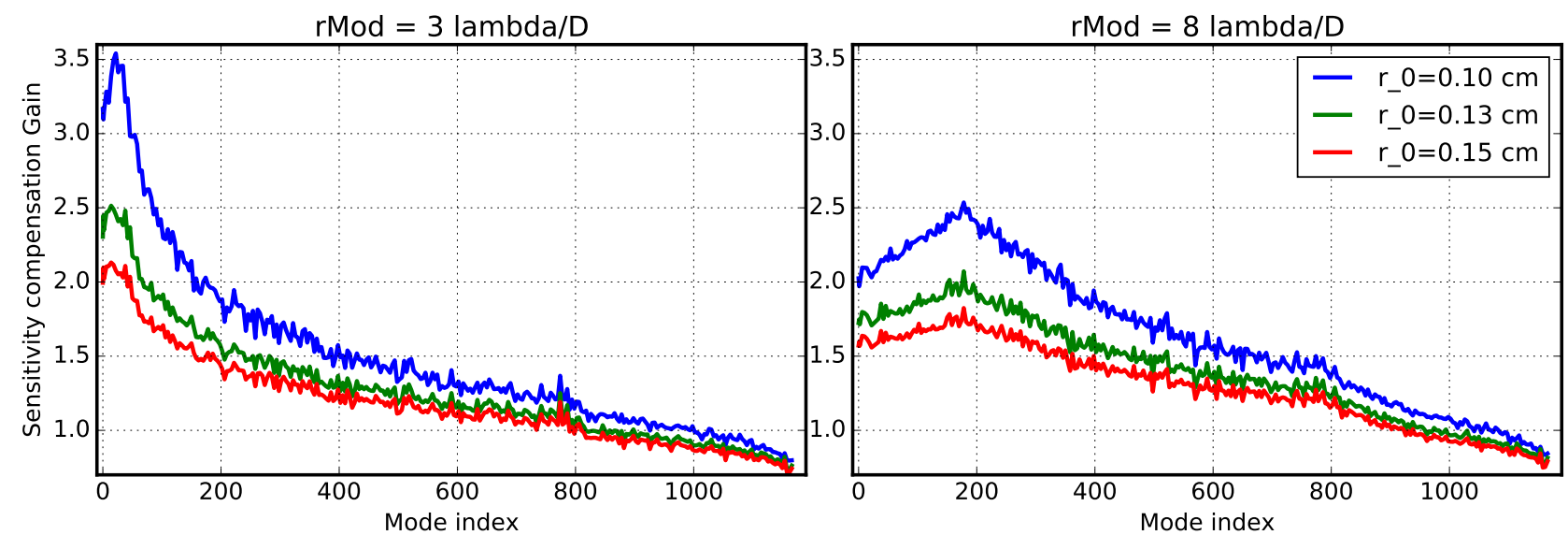

Figure 3: Optimal modal gains (a.k.a pyramid optical gains) computed for each of the controlled modes (mirror basis) on different $r_{0}$ values $(0.1,0.13$ and $0.15 \mathrm{~cm}$ ) with a modulation of $3 \lambda / D$ (left) and $8 \lambda / D$. The simulation uses here a $45 \times 45$ sup-apertures pyramid on a 18m telescope with a 40x40 DM.

The shape of the modal gains can be split in different regimes. For the lowest order modes we observe an increase of loss of sensitivity until it reaches a peak (I.e where the modal gain is the highest). This peak is reached for modes that are exactly located (at the focal plane) on the modulation radius of the pyramid. Then then loss of sensitivity decreases for higher orders modes. This behavior is explained by Verinaud ${ }^{12}$ where the Pyramid measures the derivative of the phase for modes within the modulation radius and acts as a phase sensor outside the modulation radius. When the modulation increases from 3 to $8 \lambda / D$ (from left to right on fig 3 ) the peak of loss of sensitivity is reached at higher order modes.

The difference of sensitivity between low and high orders can be greater than 3 and even higher with a strong residual of correction. This highlights the importance to calibrate this loss of sensitivity for the Pyramid especially when the PSF (at the WFS wavelength) is far from the diffraction limit. We observed indeed a much better performance and robustness of the loop, in particular when the seeing is greater than 1" using this method. The expected calibration strategy for MICADO is to evaluate the loss of sensitivity of some modes (low and high order ones) by injecting a small amplitude disturbance using the DM. Using a modulated detection from the RTC measurements we compute and update the shape of the modal gains accordingly (for all controlled modes). Of course the amplitude of the disturbance will be small enough to minimize the impact on the scientific instrument and ensure a PSF improvement. 


\section{SIMULATION RESULTS}

\subsection{SH VS Pyramid}

Goal: compare the SH and Pyramid closed loop performance using similar (and reduced) dimensioning case.

To simplify the comparison between SH and Pyramid WFS we simulated a round pupil $(\mathrm{D}=38 \mathrm{~m})$ and a $79 \times 79$ actuators squared grid. We ran a $78 \times 78$ sub-apertures SH WFS simulations using the LISA detector specifications (see Table 3) and compared the SCAO performance with a $78 \times 78$ Pyramid WFS using the ALICE detector specifications (see Table 2). To be fair with this Pyramid Vs SH comparison we also tested the case were the 2 WFSs have the same RON (3- for both). Turbulence conditions are also simplified for this test and the seeing is $0.79 "$ (with 1 layer only) and $L_{0}=25 \mathrm{~m}$. We computed the SCAO performance (SR is computed on-axis at $1.65 \mu \mathrm{m}$ ) by exploring the guide star magnitude from 10 to 18 . Results are presented in Figure 4 .

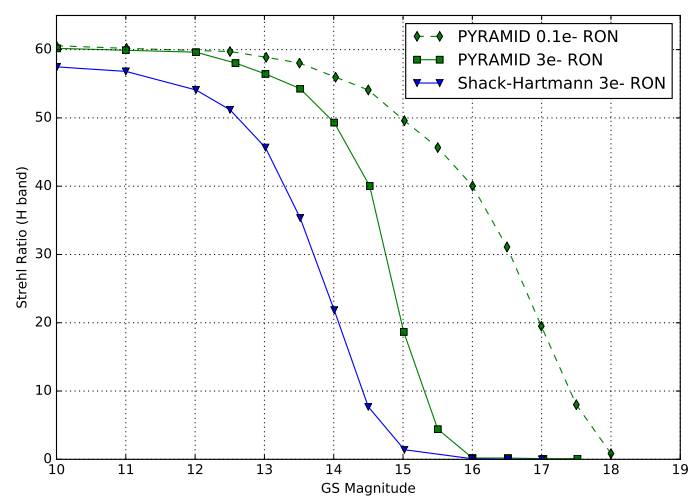

Figure 4: SCAO performance comparison (SR computed in H Band) between SH/RON3e- in blue, PYR/RON3ein green plain and PYR/RON0.1e- in green dashed.

At high SNR (mag > 11) the Pyramid WFS residuals are close to the SH (SR=60\% for PYR and $58 \%$ for $\mathrm{SH})$. In this case, for both, SCAO is mainly limited by fitting error. However at low flux since the 2 available detectors have a very different RON (0.1e- for Pyramid WFS and 3e- for SH WFS) it is not surprising to measure a much better performance for the Pyramid compared to the SH. At magnitude 15 the Pyramid (with a RON of $0.1 \mathrm{e}-$ ) still provides $50 \% \mathrm{SR}$ (green dashed curve Fig 4) while the SH is fully dominated by the detector noise giving only a $\mathrm{SR}=3 \%$ (blue line). Pyramid WFS provides a $\approx 2.5$ magnitude gain compared to $\mathrm{SH}$. In the case the Pyramid has the same detector noise penalty (3e-), Pyramid still surclasses the SH with a $\approx 1$ magnitude improvement (plain green line).

In conclusion of this MICADO SCAO WFS choice study we show that the Pyramid clearly takes advantage of the difference of noise between the 2 WFS types. The SH WFS is strongly penalized by the availability of the detector characteristics comparable with the Pyramid solution. For the SCAO, Pyramid gives a 2.5 magnitude improvement of sky coverage, among 1 magnitude is only due to its intrinsic wavefront sensing performance. The simulations results presented in the following sections now concentrate on Pyramid WFS solution only.

\subsection{MICADO SCAO performance}

\subsubsection{On-axis correction}

Goal: present in this section the results of the MICADO-SCAO simulations using a Pyramid WFS only with a as accurate as possible E-ELT scaled simulation.

We explored the SCAO performance as a function of the guide star magnitude for each of the 5 atmospheric conditions simulated (see Table 1). We used the default simulations parameters described in section 2 and loop calibration/optimization as described in section 4.

Results for median conditions (seeing $=0.703 "$ ) are presented Figure 5. At high SNR (magnitude $>=12$ ) the SCAO gives a Strehl Ratio of $80 \%$ in K Band, $70 \%$ in H, and $55 \%$ in J Band for Median seeing conditions 
(0.703"). This gives a total wavefront error of $160 \mathrm{~nm}$ rms (including fitting error). K Band long exposure PSF is illustrated for magnitude 11 (left). We clearly recognize the hexagonal DM correction zone, the diffraction patterns due to E-ELT pupil shape and the diffraction effect on spiders. At low SNR (magnitude 17) the SCAO gives a Strehl Ratio of $48 \%$ in K Band, $28 \%$ in $\mathrm{H}$, and $11 \%$ in J Band. This gives a total SCAO wavefront error of 300nm rms. K Band long exposure PSF is illustrated for magnitude 17 (right).

Figures 6a, 6b, 6c and 6d, respectively explore the SCAO performance in J, H and K Bands for Q1, Q2, Q3 and Q4 seeing conditions.
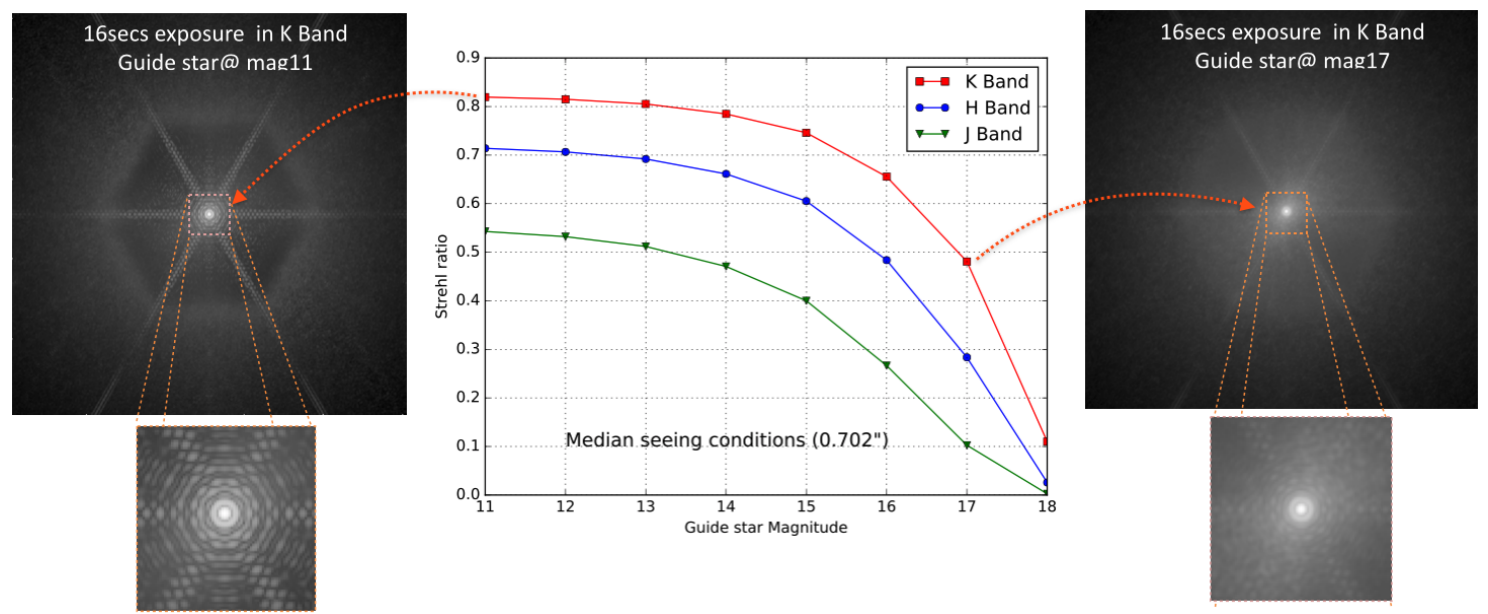

Figure 5: Strehl ratio computed on-axis using a Pyramid WFS computed in J, H, K for magnitudes from 11 to 18 for Median seeing conditions. PSFs are illustrated in K Band for magnitude 11 (left) and 17 (right).

\subsubsection{Off-axis correction}

Goal: explore the overall MICADO-SCAO performance off-axis.

We performed the off-axis exploration using the Q3 turbulence conditions only (seeing $=0.793$ "). Each PSF is computed off-axis from 0 " to 60 ", in J, H, K bands and magnitudes 11,14 and 17 . Results are presented in Figure 7. At magnitude 11 the anisoplanetism strongly degrades the performance and the SR decrease from $78 \%$ (K band) on-axis at $54 \%$ at 10 " and $26 \%$ at 20 ". At magnitude 17 the on-axis SR is $39 \%$ and only $18 \%$ at $20 "$.

It is important to be noted that the off-axis PSF are only marginally elongated even very far off-axis. This is due to outer scale value ( $25 \mathrm{~m}$ here) being much larger than the telescope diameter. Therefore the PSFs on the E-ELT even with a low SR will present a coherent core that can be used for astrometry purposes. This is a confirmation of the results already found by Clenet et al. ${ }^{13}$

\subsection{MICADO SCAO design study}

\subsubsection{Modulation radius}

Goal: Find the best Pyramid modulation radius for a given configuration.

Pyramid WFS can adapt it's own response by changing its modulation radius. A small modulation radius increases the sensitivity but lowers the linear range. Conversely, increasing the modulation radius lowers the sensitivity but increase the dynamic range. Both cases can be useful. When the amplitude of aberrations is low (good seeing, high flux...) Pyramid can work in a small amplitude regime allowing to uses small modulation radius to increase the sensitivity. However when the amplitude of perturbed wavefront increases (high AO residuals, low SNR, bad seeing...) Pyramid WFS saturates and it can be useful to increase the modulation amplitude to avoid saturation on the measurements. Pyramid WFS calibration is therefore a trade off between sensitivity and linear range of the measurements. 

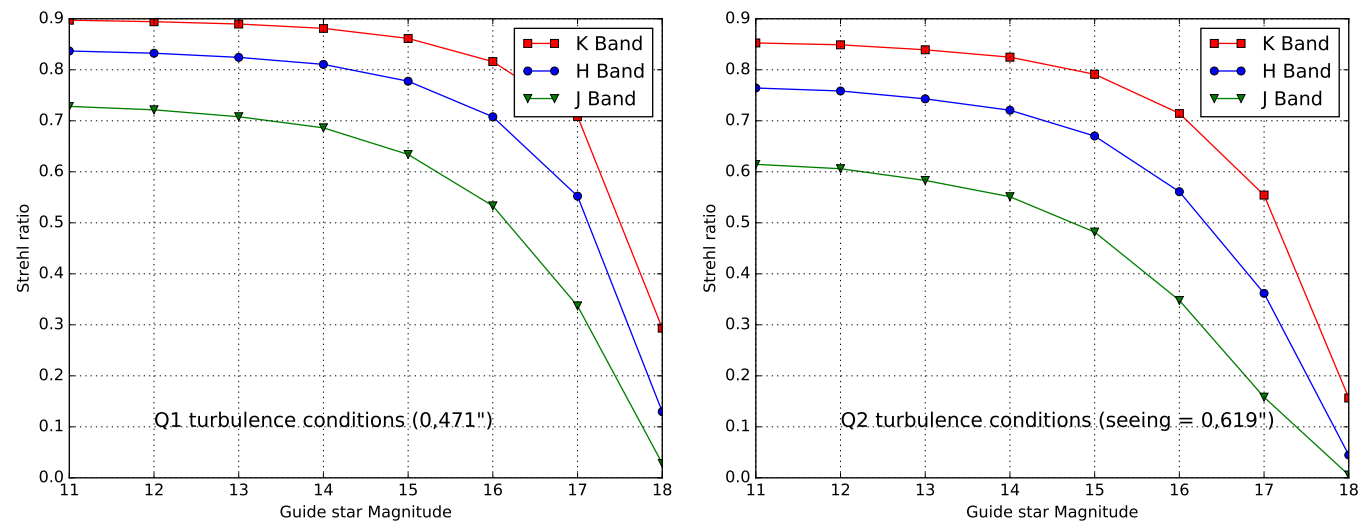

(a) Q1 Turbulence conditions (seeing $=0.471$ ")

(b) Q2 Turbulence conditions (seeing $=0.619 "$ )
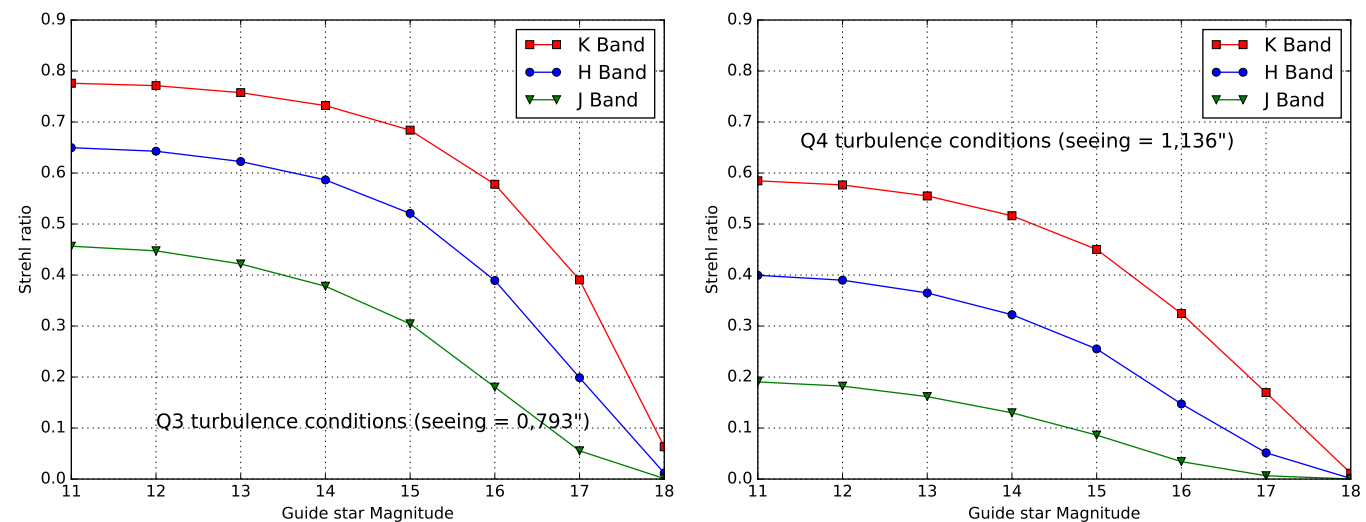

(c) Q3 Turbulence conditions (seeing $=0.793$ ")

(d) Q4 Turbulence conditions (seeing $=1.136 "$ ")

Figure 6: Strehl ratio computed on-axis using a Pyramid WFS computed in J, H, K for magnitudes from 11 to 18. Turbulence seeing conditions range from very good (Fig 6a) to bad (Fig 6d) 


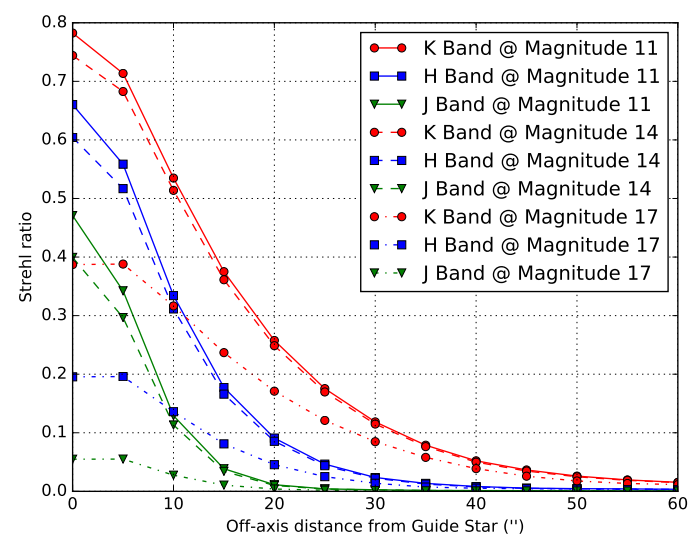

Figure 7: SR computed in J, H, K bands from 0" to 60" off-axis for guide star magnitude 11, 14 and 17 for Q3 turbulence conditions (seeing $=0.793 ")$.

We explored in this section the pyramid modulation radius between 1 and $20 \lambda / D$ using medium turbulence conditions only (Q3 - seeing 0.79"). At high SNR (mag >12) we observed that despite a very high sensitivity a very small modulation radius $(1 \lambda / D$ shown with blue circles in Fig 8$)$ leads to a worse performance than $3 \lambda / D$ (green triangles down Fig 8). Looking at the measurements we concluded that the amount of light diffracted out on each of the 4 pupils is enough to perturb the wavefront measurements on the others neighbors pupils. On the other side, a large modulation $(>10 \lambda / D)$ increase the linearity domain but the sensitivity quickly decreases compared to a moderate modulation even with high flux. In particular, we observed that the Tip-Tilt cendroiding gain is strongly affected and some modes whose sensitivity is strongly decreased can produce a almost null TipTilt on the Pyramid WFS measurments.

At low flux best results were found at modulations between 3 and $5 \lambda / D$. We note that at $1 \lambda / D$ the performance quickly decrease from magnitude 14. This is due to the Pyramid not working in its linear response range and the measurements saturates. Therefore the simulations shows that the optimal modulation radius with a median seeing is $3 \lambda / D$ for magnitude $<16$ and $5 \lambda / D$ for magnitude $>=17$.

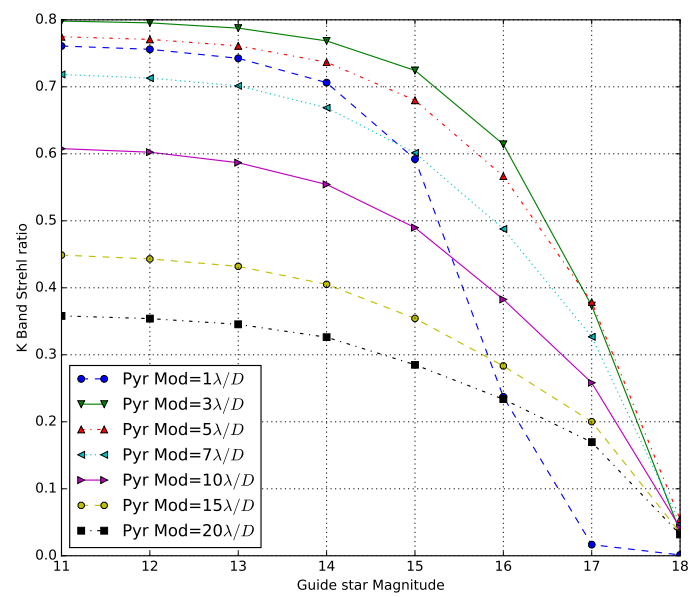

Figure 8: K Band Strehl Ratio at magnitudes 11 to 18 for Pyramid modulation radius from 1 to $20 \lambda / D$ 


\subsubsection{Pupil sampling}

Goal: Considering the fixed number of actuators defined by the M4 Deformable Mirror the trade off is to find for a given number of controlled modes the optimal number of subapertures for the Pyramid WFS sampling (no binning).

We studied the pixel sampling effect for magnitudes 11,15 and 17 . We fixed the number of controlled at 4473. Results are presented in Figure 9. At high SNR (magnitude 11) the performance is mainly limited by fitting and photon noise. Increasing the number of pixels ( $>92)$ allows to minimize aliasing effect by pushing the WFS aliased frequency further than the DM cut-off frequency. At less than 84 subapertures the performance starts to drop significantly because of the poor registration between subapertures and actuator and the number of sub-apertures becomes lower than the number of actuators.

For $m a g<=15$, the optimal and most robust value seems to be centered at 92 sub-apertures. At low SNR the optimal value is 88 sub-apertures. Next step will be to find the optimal configuration for low SNR cases and find the optimal trade-off between binning of pixels, AO loop frequency and number of spatially controlled modes. This study is still on progress and will define the MICADO-SCAO configuration modes used at the telescope.

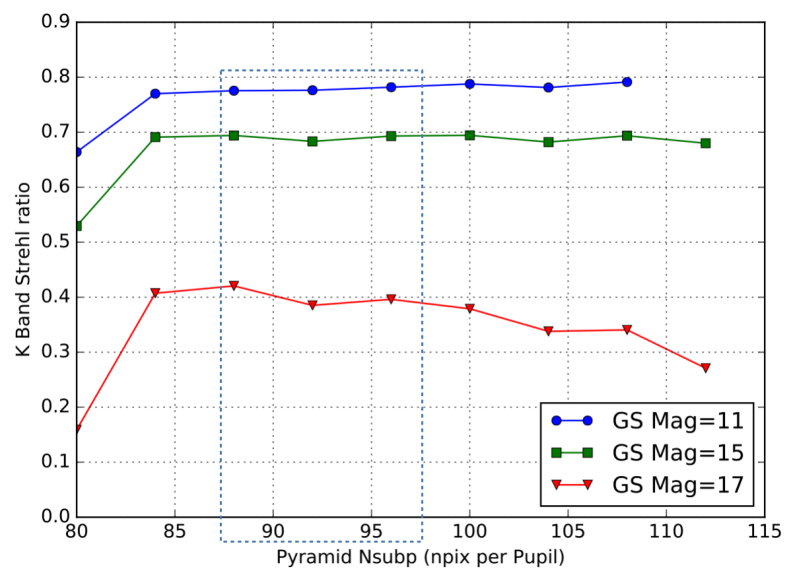

Figure 9: K Band Strehl Ratio at magnitudes 11, 15 and 17 for Pyramid pupil sampling ranging from 80 to 112 sub-apertures.

\subsubsection{SCAO performance Vs number of controlled modes}

Goal: Explore the optimal number of controlled modes for a given configuration.

We studied the number of controlled modes effect for magnitudes 11, 15 and 17. We fixed the number of sub-apertures at 92. Results are presented Figure 10. We show that it is possible to keep more than $95 \%$ of the total available modes to optimize the PSF. The optimal number of filtered modes is $\approx 100$ at magnitude $<15$ and $\approx 500$ modes with magnitude> 15 (respectively 4373 and 3973 of effective controlled modes during close loop operation).

\section{CONCLUSION}

We performed a full scale E-ELT SCAO end to end numerical simulation by following the MICADO instrument specifications. Shack-Hartmann and Pyramid WFS were both simulated. We found that Pyramid WFS gives the best performance with the current MICADO-SCAO baseline parameters. We presented the expected MICADO SCAO overall performance (on-axis and off-axis) using Pyramid WFS for J, H and K Bands and magnitude between 11 and 18. We explored some of the dedicated Pyramid WFS design parameters and found optimal values for pyramid modulation radius, pupil sampling and number of controlled modes. Future work will address the specific problems due to E-ELT configuration (differential piston effect/Island, vibrations, wind shake disturbances, pupil tracking). 


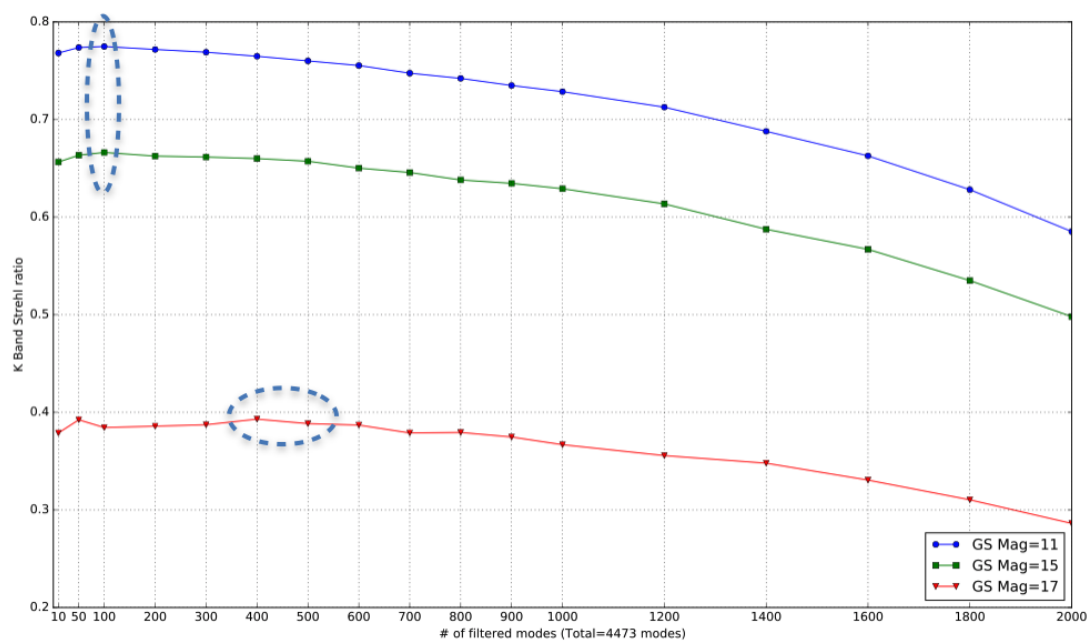

Figure 10: Pyr Nb of optimal controlled modes Vs magnitude.

\section{REFERENCES}

[1] Davies, R., Schubert, J., Hartl, M., Alves, J., Clénet, Y. et al., "MICADO: first light imager for the E-ELT", Proceedings of the SPIE, Volume 9908, id. $99081 Z 12$ pp. (2016)

[2] Diolaiti E., Ciliegi, P., Abicca, R., Agapito, G., Arcidiacono, C et al., "MAORY: adaptive optics module for the E-ELT" Proceedings of the SPIE, Volume 9909, id. 99092D 7 pp. (2016)

[3] Vernet, E., Cayrel, M., Hubin, N. Mueller, M. Biasi, R., "Specifications and design of the E-ELT M4 adaptive unit", Proceedings of the SPIE, Volume 8447, article id. 844761, 8 pp. (2012)

[4] Neichel, B. et al., "The Adaptive Optics modes for HARMONI - From Classical to Laser Assisted Tomographic AO", this conference.

[5] Schwartz N., "Sensing and control of segmented mirrors with a Pyramid wavefront sensor", This conference.

[6] R. Ragazzoni and J. Farinato, "Sensitivity of a pyramidic Wave Front sensor in closed loop Adaptive Optics", Astron. \& Astrophys. 350, L23-L26 (1999)

[7] V. Viotto, R. Ragazzoni, M. Bergomi, D. Magrin, and J. Farinato, "Expected gain in the pyramid wavefront sensor with limited Strehl ratio", A\&A 593, A100 (2016)

[8] Gach, J.-L., Feautrier, P., Balard, P., Guillaume, C. Stadler, E., "OCAM2S: an integral shutter ultrafast and low noise wavefront sensor camera for laser guide stars adaptive optics systems", Proceedings of the SPIE, Volume 9148, id. 91481910 pp. (2014)

[9] Downing, M., Kolb, J., Balard, P., Dierickx, B., Defernez, A., "LGSD/NGSD: high speed optical CMOS imagers for E-ELT adaptive optics", Proceedings of the SPIE, Volume 9154, id. 91540Q 8 pp. (2014)

[10] Korkiakoski, V. , Vérinaud, C and Le Louarn, M., "Improving the performance of a pyramid wavefront sensor with modal sensitivity compensation", Applied Optics, Vol. 41, 1, pp 79-87 (2008)

[11] Gratadour, D. , Ferreira, F., Sevin, A., et al., "COMPASS: status update and long term development plan", Proc. SPIE 9909, Adaptive Optics Systems V, 990971 (2016)

[12] Verinaud, C., "On the nature of the measurements provided by a pyramid wave-front sensor", Optics Communications, Volume 233, Issue 1-3, p. 27-38 (2004)

[13] Clénet, Y., Gendron, E., Gratadour, D., Rousset, G., Vidal, F., "Anisoplanatism effect on the E-ELT SCAO point spread function. A preserved coherent core across the field", Astronomy \& Astrophysics, Volume 583, id.A102, 10 pp. 